\title{
Aproximaciones conceptuales para la definición de un índice de especialización turística aplicado a los departamentos de Argentina
}

\author{
Conceptual approaches for the definition of a tourism specialization index \\ applied to the departments of Argentina
}

\author{
Matías Adrián Gordziejczuk \\ matiadrigord@gmail.com \\ CONICET. Grupo de Estudios Sobre Población y \\ Territorio (GESPyT), Instituto de Humanidades \\ y Ciencias Sociales (INHUS), Facultad de \\ Humanidades, Universidad Nacional de Mar del Plata., \\ Argentina
}

Recepción: 29 Septiembre 2020

Aprobación: 01 Marzo 202

Publicación: 03 Mayo 2021

Cita sugerida: Gordziejczuk, M. A.(2021).

Aproximaciones conceptuales para la definición de un índice de especialización turística aplicado a los departamentos de Argentina. Geograficando, 17(1), e092. https://doi.org/10.24215/2346898Xe092

\begin{abstract}
Resumen: El objetivo del artículo es dar a conocer un índice de especialización turística de carácter multidimensional aplicado sobre Argentina a escala departamental. Para ello se despliega una metodología de naturaleza cuantitativa vinculada al análisis espacial multivariado, el cual requiere de técnicas como la estandarización de indicadores y el cálculo de puntajes de clasificación espacial. La distribución espacial de la medida resumen obtenida se analiza junto con la exposición del ranking de especialización turística departamental. Los resultados contribuyen a subrayar ideas y aportes que vienen siendo formulados desde hace años acerca de la fragmentación territorial del turismo en Argentina, por lo menos hasta los tiempos previos al desencadenamiento de la pandemia por el covid-19.
\end{abstract}

Palabras clave: Índice de especialización turística, Departamentos y partidos, Distribución espacial, Indicadores componentes.

\begin{abstract}
The aim of the article is to present a multidimensional tourist specialization index applied to Argentina at a departmental scale. To this end, a quantitative methodology linked to multivariate spatial analysis, requiring such techniques as indicator standardization and spatial classification score calculation, is employed. Spatial distribution of the summary measure obtained is analyzed together with the exposition of the departmental tourism specialization ranking. The results help to highlight ideas and contributions that have been long formulated on the topic of territorial fragmentation of tourism in Argentina, at least before the COVID-19 pandemic outbreak.
\end{abstract}

Keywords: Tourism specialization index, Subprovincial districts, Space distribution, Component indicators.

\section{INTRODUCCIÓN}

El objetivo de este artículo es dar a conocer un índice de especialización turística (IET) de carácter multidimensional aplicado para Argentina a escala departamental. Se parte de considerar que el turismo es un fenómeno complejo que requiere ser abordado desde un enfoque multidimensional, y que muchas 
medidas que se han propuesto para evaluar la especialización turística de los espacios geográficos tienen la particularidad de tomar en cuenta para su medición una sola variable.

El trabajo conserva un espíritu exploratorio y de aplicación práctica. Básicamente se ha creado, recopilado y organizado un conjunto de datos geoestadísticos procedentes de diversas fuentes para obtener una mirada general sobre la distribución espacial de la especialización turística hacia el interior de Argentina. El equilibrio entre la intención de trabajar con un nivel de desagregación espacial mayor a la escala provincial y la posibilidad de obtener y generar datos causó que se considere a los departamentos (partidos, en el caso de la provincia de Buenos Aires) como universo de observación, sumando como una unidad espacial más a la Capital Federal.

El artículo se encuentra organizado de la siguiente manera. A continuación, se desarrollan los aspectos metodológicos que conciernen a la investigación; se presenta por un lado al IET desde un punto de vista general, contemplando las características de esta medida y los antecedentes hallados en relación a su uso, y por otro a partir de las dimensiones y los indicadores que forman parte de este índice. Luego se hace referencia a los procedimientos implementados para la obtención de puntajes de clasificación espacial, sobre la cual se adelanta su definición como una "metodología de análisis multivariado simple que se basa en la estandarización de variables con la finalidad de obtener un valor resumen” (Buzai, 2014, p. 165), que luego puede mapearse y analizarse a partir de su distribución espacial. Esto da paso a la sección de resultados, donde se exhiben la expresión cartográfica del IET, el ranking turístico departamental, y además se incorpora su descripción trenzada a lo demográfico y regional. Por último, se hallan las conclusiones.

\section{Aspectos Metodológicos}

En términos generales, para este trabajo se han tenido en cuenta las indicaciones de Schuschny y Soto (2009) sobre el diseño de indicadores compuestos, como así también las implementaciones metodológicas de naturaleza cuantitativa explicadas y aplicadas por los geógrafos Buzai (2014), Lucero (2016) y Velázquez (2016). La elaboración del IET ha requerido de las siguientes etapas: 1) justificación de la construcción y denominación del índice (fase teórico-conceptual), 2) búsqueda, selección y generación de los indicadores componentes, o lo que sería, en palabras de Buzai (2014), la creación de una matriz de datos originales, 3) análisis exploratorio de los indicadores componentes en entorno SIG de acceso libre para evaluar si efectivamente los datos seleccionados concordaban con la idea de origen (proceso de validación primaria), 4) estandarización de los indicadores componentes o pasaje a una matriz de datos estandarizados a partir del cálculo de puntajes omegas (Buzai, 2014), y 5) cálculo del IET mediante el promedio simple de los indicadores estandarizados y elaboración de la cartografía temática. En los siguientes subapartados se profundiza en torno a estas instancias.

\section{A- Acerca del indicador compuesto Índice de Especialización Turística}

Fernández, Grill y Laumann (2011) destacan al IET como un indicador idóneo para mostrar la diferenciación entre los lugares en los que el turismo está más y menos desarrollado. Sin embargo, una dificultad con la que se encontraron estas autoras fue la de clasificar a sus unidades espaciales en función del grado de especialización turística, debido a que "no se cuenta con un indicador formal para realizar esta acción” (Fernández, Grill y Laumann, 2011, p. 113), por lo que el mismo se debe elaborar en cada caso a partir de las fuentes de información disponibles según el tipo de unidad espacial de análisis. Partiendo del IET de Brau, Lanza y Pigliaru (2007), el cual se define como el promedio de la razón entre el nivel de ingreso de divisas por turismo internacional y el PBI, las autoras precitadas desarrollaron toda una serie de adaptaciones de este índice por considerar que presenta la limitación de acotarse a un solo indicador (ingreso 
de divisas por turismo internacional), dejando de lado la idea de turismo como concepto multidimensional. Con una impronta economicista similar, Precedo Ledo y Míguez Iglesias (2007) hablan de un coeficiente de especialización turística que toma en cuenta datos sobre participación del turismo en las recaudaciones municipales de España.

Es a partir de la crítica mencionada en el párrafo anterior que Fernández, Grill y Laumann (2011) postulan pensar en el IET como un indicador compuesto, es decir, "una representación simplificada que busca resumir un concepto multidimensional en un índice simple (unidimensional)" (Schuschny y Soto, 2009, p. 13). Al utilizarse un conjunto de variables se realiza un análisis más global del turismo, y se abarcan más características que simplemente el ingreso de divisas. En este sentido, las autoras contemplan en su estudio los indicadores: contribución del PBI de la industria "Viajes y Turismo" al PBI total, razón entre el capital invertido en el sector, y las inversiones totales de la economía y porcentaje de empleados de la "industria" sobre el empleo total de la economía. Más allá de esta discusión, ni el IET propuesto por Brau, Lanza y Pigliaru (2007), ni el planteado por Fernández, Grill y Laumann (2011) podrían replicarse en este trabajo, porque al nivel de departamentos argentinos no se cuenta con los datos necesarios. Esto no impide que la expresión IET pueda ser utilizada para "determinar, por ejemplo, cuáles son -dentro de un mismo país- los destinos turísticos más desarrollados" (Fernández, Grill y Laumann, 2011).

Así como la inaccesibilidad a datos descarta la replicación de las medidas de especialización turística hasta aquí comentadas, no se desconoce la existencia de otras propuestas con las que sucede lo mismo. Tal es el caso del estudio de Vera Rebollo, Ponce Herrero, Dávila Linares y Ramón Morte (1990) enfocado sobre los municipios litorales valencianos, en el que se incluyen indicadores que hacen imprescindible el trabajo de campo y el análisis desde el conocimiento empírico del territorio, como número de comercios, existencia de comunidades de residentes extranjeros y destinación de los presupuestos municipales.

Otras propuestas presentan la limitación ya mencionada de estar circunscriptas a un solo indicador. Aquí se debe hacer referencia al aporte de Defert (1967), señalado por Callizo Soneiro (1991), que consiste en la formulación de la tasa de función turística, uno de los indicadores más frecuentemente aplicados en Geografía del Turismo y que se basa en poner en relación porcentual al número de plazas turísticas y a la población residente. Si bien este indicador "posee un alto valor indicativo del peso de la actividad turística de un determinado lugar" (Callizo Soneiro, 1991, p. 37), al aportar criterios de clasificación de los espacios de acuerdo a su grado de especialización turística, en Argentina el único operativo estadístico que proporciona datos sobre plazas turísticas es la Encuesta de Ocupación Hotelera (INDEC), cuya muestra se limita únicamente a 44 centros urbanos. Por otro lado, el descarte de la idea de "tasa" viene por su objetivo de medir la frecuencia relativa con la que aparece o sucede un hecho dentro de una población y período establecido (Páez, 2013), cuestión que tampoco se podría aplicar en este caso.

Finalmente, frente a la clasificación de indicadores compuestos, propuesta por Schuschny y Soto (2009), ha de decirse que el IET no es un indicador de desempeño, vinculado a estándares regulatorios, ni tampoco un indicador basado en criterios contables, sino más bien un indicador sinóptico, o sea, una función que busca medir un atributo o característica de las unidades analizadas a partir de una mirada contextual y que se construye a partir de la agregación de información que se considera relevante sobre el tema a describir. Dicha información agregada se da a conocer en el siguiente acápite.

\section{B- Acerca de los indicadores componentes}

Tal como apunta Lucero (2016), la construcción de una medida síntesis implica la selección de un conjunto de indicadores que serán objeto de tratamientos estadísticos e informáticos. En función de las bases de datos secundarios disponibles y los datos que pueden ser recabados y sistematizados, los indicadores que finalmente son escogidos para integrar un índice representan nada más y nada menos que el resultado de un complejo proceso de análisis y debate que incluye complacencias frustraciones por la 
diferencia entre los indicadores sugeridos por la teoría, y que se desean incluir, y los indicadores con los que efectivamente se puede trabajar a partir de las matrices de datos que existen para el tipo de unidad espacial de análisis y área de estudio contemplados.

La medida síntesis elaborada en esta oportunidad no es la excepción a lo señalado en el párrafo anterior, ya que no incluye todas las dimensiones o variables que se desean observar, tales como pueden ser modalidades turísticas, equipamientos y servicios específicos. En este sentido, se aclara que este indicador compuesto no se postula como una herramienta de seguimiento ya que las fuentes de datos empleadas no permiten que se cumpla el requisito de comparabilidad entre períodos (Schuschny y Soto, 2009). Tan solo se trata de una estrategia metodológica y exploratoria que conduce a la obtención de un valor cuantitativo representativo y simbólico de la situación contemporánea (previa a la crisis desatada por el Covid-19), y no es el reflejo de un año en particular. Esto se acepta en función de la dificultad que existe en Argentina para obtener datos geoestadísticos sobre turismo que presenten tanto contigüidad como desagregación espacial, más allá del nivel provincial (Sánchez, 2011).

Dicho esto, la Tabla 1 presenta los indicadores que integran el IET. A través de ellos se pueden divisar varios de los aspectos que, según Wallingre (2014), deben ser tenidos en cuenta para la adquisición de estatus turístico. En esta investigación, esas mismas cuestiones son tomadas en cuenta como criterios para evaluar el grado de especialización turística de los Departamentos de Argentina.

TABLA 1

Composición del IET Departamental argentino

\begin{tabular}{|c|c|c|c|}
\hline Dimensiones & Variables & Indicadores & $\begin{array}{l}\text { Fuentes de } \\
\text { datos }\end{array}$ \\
\hline \multirow[b]{2}{*}{ Alojamiento } & serviciado & \begin{tabular}{|l|} 
Razón hoteles \\
turísticos-población \\
(RHP)
\end{tabular} & \multirow[b]{2}{*}{$\begin{array}{l}\text { INDEC, } \\
\text { Censo } \\
\text { Nacional de } \\
\text { Población, } \\
\text { Hogares y } \\
\text { Viviendas } \\
2010\end{array}$} \\
\hline & residenciado & $\begin{array}{l}\text { Porcentaje de } \\
\text { viviendas que se } \\
\text { usan para } \\
\text { vacaciones, fines } \\
\text { de semana u otros } \\
\text { usos temporales } \\
\text { (\%vT) } \\
\end{array}$ & \\
\hline \multirow[b]{2}{*}{ Atractivos } & naturales & $\begin{array}{l}\text { Presencia de } \\
\text { Recursos } \\
\text { Recreativos de Base } \\
\text { Natural (RRBN) }\end{array}$ & \multirow[b]{2}{*}{$\begin{array}{l}\text { Velázquez } \\
\text { (Dir.) } 2016\end{array}$} \\
\hline & culturales & $\begin{array}{l}\text { Presencia de } \\
\text { Recursos } \\
\text { Recreativos } \\
\text { Socialmente } \\
\text { Construidos (RRSC) } \\
\end{array}$ & \\
\hline \multirow{2}{*}{$\begin{array}{l}\text { Gestión } \\
\text { turística }\end{array}$} & privada & $\begin{array}{l}\text { Razón agencias de } \\
\text { viaje-población } \\
\text { (RAP) }\end{array}$ & $\begin{array}{l}\text { Secretaria de } \\
\text { Turismo de } \\
\text { la Nación } \\
2017 \text { e } \\
\text { INDEC } 2010 \\
\end{array}$ \\
\hline & pública & $\begin{array}{l}\text { Organismo público } \\
\text { municipal de } \\
\text { gestión del turismo } \\
\text { con la jerarquía } \\
\text { más alta (OGT }>\text { ) }\end{array}$ & $\begin{array}{l}\text { Sitios web } \\
\text { oficiales y } \\
\text { Google } \\
\text { Noticias }\end{array}$ \\
\hline $\begin{array}{l}\text { Modalidad } \\
\text { turística }\end{array}$ & rural & $\begin{array}{l}\text { Porcentaje de } \\
\text { explotaciones } \\
\text { agropecuarias con } \\
\text { turismo rural } \\
\text { (\%ETR) } \\
\end{array}$ & $\begin{array}{l}\text { INDEC, } \\
\text { Censo } \\
\text { Nacional } \\
\text { Agropecuario } \\
2002 \\
\end{array}$ \\
\hline
\end{tabular}

Fuente: elaboración personal 
La búsqueda de datos y su indagación preliminar derivó en la conformación de cuatro dimensiones. Ellas son el alojamiento, los atractivos, la gestión turística y la modalidad turística. Aquí es importante recordar que la denominación de dimensiones e indicadores y la organización de los datos, si bien son tareas necesarias para acceder al conocimiento caracterizado generalmente como "objetivo", siempre contienen por detrás una carga de subjetividad basada en las decisiones que el investigador toma en relación a sus saberes previos. En los párrafos que se despliegan a continuación se presentan, definen y justifican cada una de estas dimensiones.

El alojamiento con pernoctación en un lugar distinto al de la residencia habitual es uno de los elementos comúnmente tenidos en cuenta a la hora de definir al turismo (Sancho, 1998). Mediante tal aspecto se está estudiando una parte fundamental del requisito de poseer infraestructura y equipamiento capaz de satisfacer las necesidades de los visitantes (Wallingre, 2014), refiriendo en este caso a la necesidad de albergue y resguardo. Tal como expresan Mantero, Laffan y Lefrou (2011), el tipo de alojamiento determina la variación entre lo que se conoce como turismo serviciado y turismo residenciado. Mientras que el turismo serviciado es aquel que implica la contratación del servicio de hotelería en cualquiera de sus formas y categorías, el turismo residenciado refiere a los casos en los cuales se hace uso de una vivienda, ya sea casa o departamento, para la estadía en el lugar de destino (Mantero, Laffan y Lefrou, 2011). En este sentido, en el IET se incluyen los indicadores denominados razón hoteles turísticos-población y porcentaje de viviendas que se usan para vacaciones, fines de semana u otros usos temporales, cuyos datos provienen del Censo Nacional de Población, Hogares y Viviendas (CNPHyV) 2010. Vale aclarar que en este censo, los hoteles turísticos se encuentran constituyendo la nómina de categorías que hacen a la variable tipo de vivienda colectiva. Al estar esta expresada en valores absolutos, se trabaja con un cociente para menguar el condicionamiento de la estructura urbana y hacer comparables los datos entre sí (Sánchez, 2011). Esto mismo se replica para el caso de las agencias de viajes.

Por su parte, el segundo indicador se obtiene de la variable censal condición de ocupación de la vivienda y respecto a él se remarca que ya viene siendo contemplado en trabajos como los de Velázquez (2001) y Rodríguez (2011) como "una medida indirecta del grado de atracción que ejercen los departamentos localizados en un paisaje determinado" (Velázquez, 2001:181) puesto que habla de las áreas elegidas por la población para su esparcimiento y sus vacaciones (Rodríguez, 2011). Este indicador se obtiene de calcular el porcentaje entre el total de viviendas particulares existentes en cada unidad espacial y el total de viviendas particulares que, según el INDEC, "se utiliza bien en períodos de descanso (fin de semana, vacaciones) o bien en otro uso temporario (alquiler a extranjeros, viajes de trabajo). Por ejemplo: casa quinta, viviendas en country, en clubes, en zonas de veraneo o vacaciones de invierno" (INDEC 2013,3).

Sobre los atractivos turísticos, Bertoncello (2008) explica que son rasgos, características o atributos naturales y culturales presentes en los lugares de destino, resultantes de un proceso de asignación de valoraciones positivas basadas en cuestiones estéticas o emocionales y que incitan el desplazamiento por el interés en conocerlos y disfrutar de ellos. Esta dimensión responde al requerimiento de que los Departamentos dispongan de recursos capaces de ser convertidos en productos turísticos (Wallingre, 2014). En esta oportunidad, se han tenido en cuenta dos indicadores provenientes de la obra de Velázquez (2016), cuyo anexo estadístico cuenta con los puntajes asignados a cada Departamento del país en base a la evaluación de la presencia de recursos recreativos de base natural (RRBN) y presencia de recursos recreativos socialmente construidos (RRSC). No se trata de indicadores que contabilizan atractivos turísticos, sino de medidas que, indirectamente, se asocian a esta dimensión porque refieren a los espacios de ocio y recreación que no solo son utilizados por los residentes sino también por los turistas (Velázquez y Celemín, 2013). Si bien dichas expresiones aparecen originalmente en Velázquez y Celemín (2013), las mismas han sido recuperadas y utilizadas en investigaciones posteriores, como las de Tapia, Quiroga y Sánchez (2015) y Velázquez (2016). Específicamente, se trata de valoraciones cuantitativas construidas bajo un criterio de evaluación subjetivo, es decir, apreciaciones debatidas y acordadas por los autores; apoyadas tanto en fuentes secundarias como 
en el conocimiento directo del terreno. Mientras que la categoría RRBN reúne elementos de esparcimiento como playas, balnearios a orillas de ríos, lagos, lagunas o diques, centros termales y de nieve, geoformas de valor escénico y recreativo, espejos y cursos de agua, y espacios verdes; la categoría RRSC tiene en cuenta a los edificios públicos y privados de valor patrimonial y a los centros culturales, comerciales y deportivos (Cfr. Velázquez y Celemín, 2013).

Respecto a la tercera dimensión, Marchena Gómez (2015) reconoce a la gestión turística como un aspecto clave para alcanzar el desarrollo sostenible y competitivo en los lugares de destino. Según Wallingre (2014), para estimular el turismo y su gestión a nivel local se requiere de figuras privadas y públicas que, por un lado, promocionen y regulen la actividad, y por otro, formulen y planifiquen proyectos o políticas. En este sentido, esta dimensión apunta a identificar el 'peso' que tienen en cada unidad espacial los actores sociales con mayor grado de responsabilidad en el despliegue del turismo, considerando las vertientes privada y pública. Puesto que disponer de empresas productoras de servicios turísticos también es uno de los requisitos que Wallingre (2014) propone para la corroboración del estatus turístico, en representación del sector privado se añade al IET el indicador llamado razón agencias de viaje- población, el cual se obtiene a partir del cruce entre los datos del Registro de agencias de viajes publicado por la de aquel entonces Secretaría de Turismo de la Nación (2017) y el CNPHyV (2010). Si bien el Registro mencionado sí cuenta con la clasificación de las agencias entre empresas de viajes y turismo, agencias de turismo, agencias de pasajes y entidades no mercantiles sin fines de lucro, se ha optado trabajar con la sumatoria ya que todas estas categorías tienen como denominador común la puesta a disposición del público de productos turísticos. Vale tener en cuenta que, pese al avance de la desintermediación de los vínculos comerciales asociados al turismo como consecuencia del acceso masivo a internet y el crecimiento de las formas de turismo alternativo, las agencias de viaje siguen siendo importantes y necesarias para muchos sectores de la población como proveedoras de reputación, confianza, información y atención (Naradowski, Remes Lenicov y Mallamace, 2014).

En lo que respecta al sector público, mediante búsquedas personales en sitios webs se ha podido construir el indicador denominado organismo público municipal de gestión del turismo con la jerarquía más alta. La información se ha extraído de los organigramas oficiales y Google Noticias, aprovechándose en este último caso que los gobiernos locales suelen difundir en los medios de comunicación los proyectos, programas, planes, iniciativas y procesos de puesta en valor que ayudan a fomentar, promover o estimular la actividad turística. Estudios como los de Benseny (2006) y Beier (2015) ya han trabajado con esta variable, alegando que ella expresa el nivel de compromiso de los gobiernos locales con la gestión del turismo. Respecto a las categorías de esta variable, mediante la lectura del trabajo de Benseny (2006) se ha podido identificar que la nómina de niveles jerárquicos se compone de mayor a menor de: Ente, Secretaría, Subsecretaría, Dirección, Subdirección, Departamento, División, Coordinación, Comisión y Consejo. Esto guarda coherencia con las apreciaciones de Beier (2015), quien postula que, al tiempo que la existencia de una Dirección de Turismo implicaría un compromiso medio con la actividad, la mera existencia de una oficina de informes no es suficiente para reflejar el nivel de empeño que demanda la actividad turística en un lugar. Teniendo en mente estas apreciaciones, se le ha asignado a cada Departamento un valor cuantitativo en función de la máxima jerarquía hallada.

Finalmente, la posibilidad de acrecentar la cantidad de indicadores componentes del IET a partir de datos emanados de las estadísticas oficiales derivó en la consideración de la modalidad de turismo rural como un cuarto aspecto a contemplar. En relación a este concepto, Cazes (2002) lo encuadra dentro del turismo alternativo que engloba a las iniciativas turísticas que se caracterizan por la búsqueda de originalidad y el deseo de evadir las rutas del turismo popular. Se trata de una reacción ética, ideológica y política de rechazo al turismo de masas, que cobró fuerza a partir de las décadas de 1970 y 1980, en concordancia con la difusión de los principios del desarrollo sostenible y la preservación de las tradiciones; hechos que, desde el punto de vista turístico, ocasionaron una atracción hacia formas de acogida que están más en sintonía con lo típico del lugar y vínculos más personalizados entre anfitriones y turistas (Cazes, 2002). En relación 
a ello, el turismo rural se inscribe dentro de la modalidad de turismo alternativo, contribuyendo además a la diversificación, pluriactividad y revitalización de los territorios rurales (Nogar y Jacinto, 2010). Por todo esto se incluye el indicador porcentaje de explotaciones agropecuarias con turismo rural, cuyos datos provienen de los resultados sobre actividades con fines comerciales conexas a la agricultura, ganadería y explotación forestal informadas en el Censo Nacional Agropecuario (CNA) 2002. Vale aclarar que si bien se han realizado censos agropecuarios en 2008 y 2018, en el primer caso no se han publicado los datos sobre turismo rural y en el segundo caso no se han difundido, hasta el momento de redacción de este artículo, los resultados a nivel de Departamentos.

\section{C- La estandarización de variables y los puntajes omegas}

Como ya se ha dicho, un índice o indicador compuesto refiere a toda aquella medida cuantitativa que combina indicadores asociados a un mismo concepto (Lazarsfeld, 1973, en Cea D’Ancona, 1996). Si bien por un lado tienen la capacidad de informar de manera sencilla al público en general y servir como herramienta para la gestión política y económica, también se debe aclarar que al simplificarse grandes volúmenes de datos se reduce el poder de análisis (Lucero, 2016).

Hacer comparables los datos que proceden de diversas fuentes y refieren a distintas unidades de cuenta, llevándolos a una misma unidad de medida, es un paso fundamental para la obtención de un índice. Este procedimiento, denominado estandarización de variables, inicia con el diseño de una matriz compuesta por filas y columnas que contienen, respectivamente, a la totalidad de las unidades espaciales e indicadores considerados en una investigación, continua con el pasaje a una segunda matriz en la que se relativizan los valores absolutos de la primera para trabajar en base a porcentajes, y concluye con una tercera matriz que provee los datos que finalmente permiten calcular el índice y mapearlo (Buzai, 2014; Lucero, 2016).

Siguiendo la metodología de Buzai (2014), en esta ocasión el proceso de estandarización o normalización de los datos se efectuó mediante el cálculo de puntajes omegas, en donde los valores originales de cada indicador son llevados a un rango de 0 a 1, y luego multiplicados por 10 para hacer más familiar su comprensión. La fórmula que se detalla a continuación se ha aplicado a cada uno de los valores contenidos en la segunda matriz y los resultados de esto componen la tercera matriz:

$$
\omega=\frac{x_{i-m}}{\mathrm{M}-m}
$$

$\omega$ es el puntaje estándar del i-esimo dato, $x_{i}$ es el dato original a ser estandarizado, $m$ es el menor valor del indicador y $\mathrm{M}$ es el mayor valor del indicador.

Cumplidos estos pasos, el IET de cada unidad espacial se obtiene al calcular el promedio entre los puntajes omega de los indicadores. Mediante el mismo procedimiento se pueden obtener sub-índices según dimensiones y, además, asignar ponderaciones o pesos diferenciales tanto a las dimensiones del índice final como a los indicadores de los sub-índices de acuerdo al criterio del investigador. A continuación se pasa a los resultados.

\section{Resultados}

La Figura 1 muestra la distribución espacial del IET. Para su representación se siguió la recomendación de Buzai (2014) de aplicar cinco intervalos de clase que permitan apreciar una situación intermedia, dos consecutivas a ella (medio alto y medio bajo) y dos situaciones extremas (alto y bajo). En esta ocasión se optó por el criterio de clasificación de cuantiles, porque al asignar a cada clase el mismo número de unidades espaciales no se dejan intervalos vacíos ni con muchas o pocas unidades, asegurándose labores futuras de comparación con otros índices o indicadores. Vale aclarar que esto no impidió que Distritos con 
valores muy diferentes entre sí queden dentro de una misma categoría, tal como ocurrió con el grupo de Departamentos con IET alto. Esta distorsión se sobrelleva en esta oportunidad con el acompañamiento de elementos complementarios al mapa tales como las tablas 2, 3, 4 y 5 .

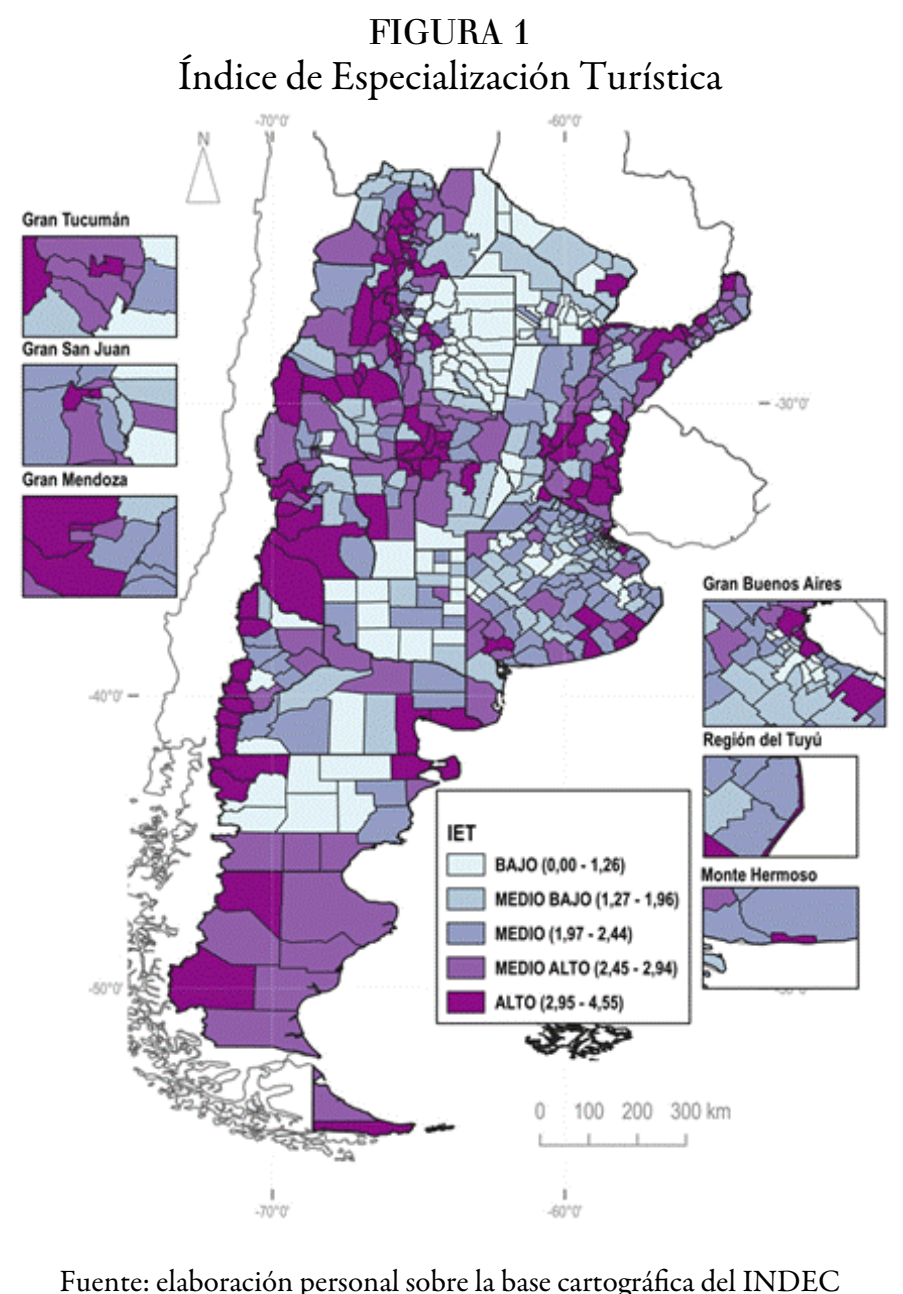

El IET adquiere una distribución espacial muy atomizada y cada una de las categorías se esparce por las distintas regiones del país, revistiendo a todas ellas de cierta heterogeneidad. Esta dispersión de los diferentes grados de especialización turística conduce a reconocer la expansión territorial del turismo en la Argentina, y para esto hay que tener en cuenta factores referidos por Bertoncello (2006) como la fragmentación del tiempo de ocio y del consumo, el surgimiento de nichos específicos en el mercado y la creciente competencia entre los lugares. En este sentido, el mayor nivel de especialización turística encierra por sí solo situaciones muy disímiles, que van desde lugares con una gran participación de turismo externo, como pueden ser Capital Federal, Lago Argentino y Bariloche, pasando por sitios de relevancia turística a nivel nacional (por ejemplo General Pueyrredón); hasta unidades espaciales cuya importancia turística se explica desde un contexto regional o provincial.

Por su parte, la Tabla 2 exhibe los extremos del ranking, los cuales varían entre los 4,5 puntos obtenidos por el Partido de Pinamar (Buenos Aires) y los 0,2 puntos de Limay Mahuida (La Pampa). Es importante resaltar que los indicadores puestos bajo tratamiento estadístico en esta oportunidad determinaron valores relativamente bajos para todo el universo de observación y que mediante el acceso a otros datos se podrían alcanzar puntajes más altos sin que ello signifique una modificación sustancial en el orden de las unidades espaciales. 
TABLA 2

IET, Departamentos posicionados en los primeros y últimos lugares

\begin{tabular}{|l|l|l|l|l|l|l|l|}
\hline \multicolumn{2}{|l|}{ Primeros en el ranking } & \multicolumn{3}{l|}{ Últimos en el ranking } \\
\hline $\begin{array}{l}\text { Pue } \\
\text { sto }\end{array}$ & Provincia & Departamento & IET & Puesto & Provincia & Departamento & IET \\
\hline 1 & $\begin{array}{l}\text { Buenos } \\
\text { Aires }\end{array}$ & Pinamar & 4,55 & 487 & Formosa & Bermejo & 0,80 \\
\hline 2 & $\begin{array}{l}\text { Buenos } \\
\text { Aires }\end{array}$ & $\begin{array}{l}\text { Monte } \\
\text { Hermoso }\end{array}$ & 4,53 & 488 & $\begin{array}{l}\text { Buenos } \\
\text { Aires }\end{array}$ & $\begin{array}{l}\text { Florentino } \\
\text { Ameghino }\end{array}$ & 0,79 \\
\hline 3 & $\begin{array}{l}\text { Buenos } \\
\text { Aires }\end{array}$ & Villa Gesell & 4,49 & 489 & $\begin{array}{l}\text { La } \\
\text { Pampa }\end{array}$ & Chical Có & 0,78 \\
\hline 4 & $\begin{array}{l}\text { Buenos } \\
\text { Aires }\end{array}$ & La Costa & 4,41 & 490 & Formosa & Ramón Lista & 0,78 \\
\hline 5 & $\begin{array}{l}\text { Buenos } \\
\text { Aires }\end{array}$ & $\begin{array}{l}\text { General } \\
\text { Pueyrredón }\end{array}$ & 4,29 & 491 & $\begin{array}{l}\text { Santiago } \\
\text { del } \\
\text { Estero }\end{array}$ & Copo & 0,77 \\
\hline 6 & Neuquén & Los Lagos & 4,15 & 492 & $\begin{array}{l}\text { Buenos } \\
\text { Aires }\end{array}$ & Tres Lomas & 0,77 \\
\hline 7 & Córdoba & Calamuchita & 3,92 & 493 & $\begin{array}{l}\text { Santiago } \\
\text { del } \\
\text { Estero }\end{array}$ & Belgrano & 0,76 \\
\hline 9 & $\begin{array}{l}\text { Buenos } \\
\text { Aires }\end{array}$ & Necochea & 3,91 & 495 & $\begin{array}{l}\text { Buenos } \\
\text { Aires }\end{array}$ & $\begin{array}{l}\text { Hipólito } \\
\text { Yrigoyen }\end{array}$ & 0,76 \\
\hline
\end{tabular}




\begin{tabular}{|l|l|l|l|l|l|l|l|}
\hline 10 & $\begin{array}{l}\text { Buenos } \\
\text { Aires }\end{array}$ & $\begin{array}{l}\text { General } \\
\text { Alvarado }\end{array}$ & 3,88 & 496 & Chaco & Tapenagá & 0,75 \\
\hline 11 & $\begin{array}{l}\text { Rio } \\
\text { Negro }\end{array}$ & Bariloche & 3,87 & 497 & $\begin{array}{l}\text { La } \\
\text { Pampa }\end{array}$ & Chalileo & 0,70 \\
\hline 12 & Córdoba & San Alberto & 3,79 & 498 & $\begin{array}{l}\text { Santiago } \\
\text { del } \\
\text { Estero }\end{array}$ & Alberdi & 0,69 \\
\hline 13 & $\begin{array}{l}\text { Tierra } \\
\text { del } \\
\text { Fuego }\end{array}$ & Ushuaia & 3,78 & 499 & $\begin{array}{l}\text { Santiago } \\
\text { del } \\
\text { Estero }\end{array}$ & Sarmiento & 0,68 \\
\hline 14 & $\begin{array}{l}\text { Santa } \\
\text { Cruz }\end{array}$ & $\begin{array}{l}\text { Lago } \\
\text { Argentino }\end{array}$ & 3,77 & 500 & $\begin{array}{l}\text { Santiago } \\
\text { del } \\
\text { Estero }\end{array}$ & Jiménez & 0,68 \\
\hline 15 & La Rioja & Sanagasta & 3,77 & 501 & $\begin{array}{l}\text { Santiago } \\
\text { del } \\
\text { Estero }\end{array}$ & Avellaneda & 0,67 \\
\hline 16 & Neuquén & Lacar & 3,74 & 502 & $\begin{array}{l}\text { La } \\
\text { Pampa }\end{array}$ & Lihuel Calel & 0,65 \\
\hline 17 & $\begin{array}{l}\text { Catamar } \\
\text { ca }\end{array}$ & Ambato & 3,72 & 503 & $\begin{array}{l}\text { Buenos } \\
\text { Aires }\end{array}$ & Salliqueló & 0,64 \\
\hline 18 & Salta & Capital & 3,70 & 504 & $\begin{array}{l}\text { La } \\
\text { Pampa }\end{array}$ & Curacó & 0,62 \\
\hline 19 & San Luis & Junín & 3,69 & 505 & $\begin{array}{l}\text { Rio } \\
\text { Negro }\end{array}$ & 9 de Julio & 0,61 \\
\hline
\end{tabular}




\begin{tabular}{|l|l|l|l|l|l|l|l|}
\hline 20 & Mendoza & Capital & 3,69 & 506 & $\begin{array}{l}\text { Santiago } \\
\text { del } \\
\text { Estero }\end{array}$ & Silipica & 0,61 \\
\hline 21 & Tucumán & Tafi del Valle & 3,67 & 507 & $\begin{array}{l}\text { Santiago } \\
\text { del } \\
\text { Estero }\end{array}$ & Mitre & 0,59 \\
\hline 22 & Jujuy & $\begin{array}{l}\text { Dr. Manuel } \\
\text { Belgrano }\end{array}$ & 3,63 & 508 & $\begin{array}{l}\text { Santiago } \\
\text { del } \\
\text { Estero }\end{array}$ & Figueroa & 0,59 \\
\hline 23 & Córdoba & Santa Maria & 3,62 & 509 & Salta & Rivadavia & 0,58 \\
\hline 24 & Chubut & Futaleufú & 3,62 & 510 & $\begin{array}{l}\text { Río } \\
\text { Negro }\end{array}$ & Ñorquinco & 0,48 \\
\hline 25 & Misiones & Iguazú & 3,59 & 511 & $\begin{array}{l}\text { La } \\
\text { Pampa }\end{array}$ & Limay Mahuida & 0,26 \\
\hline
\end{tabular}

Fuente: elaboración personal

Por otro lado, las Tablas 3, 4 y 5 se incorporan con la intensión de caracterizar las categorías del IET. En el primer caso se observan los valores absolutos y relativos de población urbana, rural, rural agrupada y rural dispersa. Lo primero que resalta es la considerable diferencia entre el volumen demográfico de los Departamentos con el nivel de especialización turística más alto y el resto de las categorías, las cuales se reparten volúmenes inferiores de población pero no demasiado disímiles entre sí. Traducido a números porcentuales, mientras que el 38,7\% de los argentinos censados en 2010 residían en Departamentos catalogados con un IET alto, los otros intervalos concentraban cada uno entre un 17,7 y $12,9 \%$.

La distribución de la población en cada una de las categorías del IET es semejante al comportamiento nacional, puesto que se aprecia una amplia predominancia de la población urbana sobre la rural y, a su vez, una superioridad del volumen de personas que residen en hábitats rurales dispersos por sobre los que habitan en hábitats rurales agrupados. Entre algunas singularidades se destaca que, por ejemplo, el peso de la población urbana desciende junto con el IET, a excepción del último rango donde se registra un aumento. Aquí hay que tener en cuenta la influencia que ejercen Partidos del oeste del Conurbano bonaerense que son totalmente urbanos y que han obtenido IET's bajos. Otro aspecto a resaltar es que, mientras la población rural agrupada tiene su mayor presencia en el intervalo más alto del IET, contrariamente la población rural dispersa alcanza su porcentaje más elevado en el intervalo más bajo. La concentración de población, capitales, servicios e infraestructuras que demanda el turismo puede que contribuya a explicar el motivo de esta diferencia.

Si bien no sería correcto responsabilizar únicamente al turismo por la forma en que se distribuyen los datos de las variables contenidas en la Tabla 3, tampoco hay que descuidar que "la función urbana que más se asocia a altas tasas de crecimiento es la turística [y que] los centros turísticos terminan ejerciendo una fuerza centrípeta que absorbe población" (Sánchez, 2011:41-42), a raíz de cuestiones como la demanda intensiva de mano de obra (Fernández, Grill y Laumann, 2011) y las migraciones motivadas por las amenidades de las que gozan estos lugares (González, Otero, Nakayama y Marioni, 2009). Aspectos como estos deben ser tenidos en cuenta dado que contribuyen a comprender el motivo de tales diferencias demográficas entre las categorías del IET. 
TABLA 3

Población según categorías del IET

\begin{tabular}{|c|c|c|c|c|c|c|c|c|c|c|}
\hline & \multicolumn{9}{|l|}{ Población } \\
\hline & & Urbana & (\%) & Rural & (\%) & $\begin{array}{l}\text { Rural } \\
\text { Agrupada }\end{array}$ & $(\%)$ & $\begin{array}{l}\text { Rural } \\
\text { Dispersa }\end{array}$ & (\%) & Total \\
\hline \multirow{5}{*}{ IET } & Alto & 14.726 .545 & 94,8 & 794.677 & 5,1 & 317.073 & 39,9 & 477.604 & 60,1 & 15.521 .222 \\
\hline & $\begin{array}{l}\text { Medio } \\
\text { Alto }\end{array}$ & 6.235 .750 & 87,6 & 879.954 & 12,3 & 298.441 & 33,9 & 581.513 & 66,0 & 7.115 .704 \\
\hline & Medio & 4.970 .195 & 87,3 & 721.412 & 12,6 & 266.910 & 37,0 & 454.502 & 63,0 & 5.691 .607 \\
\hline & $\begin{array}{l}\text { Medio } \\
\text { Bajo }\end{array}$ & 4.490 .756 & 86,7 & 688.766 & 13,3 & 254.059 & 36,8 & 434.707 & 63,1 & 5.179 .522 \\
\hline & Bajo & 6.043 .999 & 91,4 & 564.835 & 8,5 & 182.555 & 32,3 & 382.280 & 67,6 & 6.608 .834 \\
\hline
\end{tabular}

Fuente: elaboración personal con base en datos del CNPHyV 2010

Por su parte, la Tabla 4 muestra el promedio que obtienen los indicadores del IET dentro de cada intervalo. A excepción de dos casos, conforme aumenta el nivel de especialización turística también se elevan los valores porcentuales, es decir que, en general, todos los indicadores considerados en esta ocasión atraviesan los intervalos del índice manifestando una asociación positiva. Si bien la ruptura de esta tendencia entre las categorías medio-bajo y medio del indicador RRBN es mínima, esta no lo es tanto en el caso de los intervalos medio y medio-alto del porcentaje de viviendas que se usan para vacaciones, fines de semana u otros usos temporales. Revisando en la base de datos y en el SIG se encuentra como posible explicación la mayor circunscripción de la categoría medio a sitios de radicación natural de segundas residencias como por ejemplo los espacios rurubanos que bordean a las grandes aglomeraciones y que para llegar a ellos desde los núcleos urbanos demandan desplazamientos relativamente cortos. Más allá de estas excepciones, se debe resaltar que las categorías alto y bajo mantienen en todos los casos los valores máximos y mínimos respectivamente.

TABLA 4

Promedio de los indicadores componentes del IET para cada una de sus categorías

\begin{tabular}{|c|l|l|l|l|l|l|l|l|}
\hline \multicolumn{2}{|c|}{} & \multicolumn{7}{|l|}{ Indicadores componentes (\%) } \\
\cline { 2 - 9 } & RHP & $\%$ VUT & RRBN & RRSC & RAP & OGT $>$ & $\%$ ETR \\
\hline \multirow{4}{*}{ IET } & Alto & 0,136 & 7,278 & 7,941 & 6,325 & 0,020 & $6,4^{*}$ & 1,797 \\
\cline { 2 - 8 } & $\begin{array}{l}\text { Medio } \\
\text { Alto }\end{array}$ & 0,034 & 2,000 & 6,329 & 5,148 & 0,006 & $5,8^{* *}$ & 0,875 \\
\cline { 2 - 8 } & Medio & 0,031 & 3,028 & 4,736 & 4,448 & 0,005 & $5,2^{* * *}$ & 0,387 \\
\cline { 2 - 8 } & $\begin{array}{l}\text { Medio } \\
\text { Bajo }\end{array}$ & 0,022 & 2,577 & 4,786 & 4,165 & 0,004 & $4,5^{* * * *}$ & 0,231 \\
\cline { 2 - 8 } & Bajo & 0,020 & 1,433 & 3,198 & 3,326 & 0,002 & $0,0^{* * * * *}$ & 0,178 \\
\hline
\end{tabular}

Fuente: elaboración personal

* Equivalente a una Secretaría de Turismo y (...)/ Secretaría de (...) y Turismo

${ }^{* *}$ Equivalente a una Subsecretaría de Turismo

${ }^{* * *}$ Equivalente a una Dirección de Turismo

**** Equivalente a una Dirección de Turismo y (...)/ Dirección de (...) y Turismo

***** Equivalente a Sin dato/ Sin organismo

Finalmente, la Tabla 5 revela cómo se distribuyen las categorías del IET hacia el interior de cada región argentina. En Cuyo y Patagonia más del 50\% de las unidades espaciales poseen un nivel de especialización turística alto y medio-alto, siendo quizás estas las regiones donde más se depende del turismo. Contrariamente, en la Región Pampeana dichas categorías son las que menos pesan en la representación de sus Departamentos y Partidos. Por su parte, en el NOA se aprecia como rasgo distintivo la polarización, puesto que es en los rangos extremos donde se reúnen casi el 50\% de los Distritos. En lo que respecta al NEA, 
su composición es muy particular ya que los mayores porcentajes se evidencian en los intervalos medio-alto y bajo.

Pese a lo comentado en el párrafo precedente sobre la Región Pampeana, ya se ha demostrado en la Tabla 2 que el podio del IET está totalmente apropiado por Departamentos que integran esta región, y más específicamente por Partidos de la Provincia de Buenos Aires que son destinos de sol y playa: Pinamar, Monte Hermoso y Villa Gesell, seguidos en cuarta y quinta posición por los Partidos de La Costa y General Pueyrredón. Salvo este último, el resto comparten las características de poseer una reducida extensión territorial y estructura básicamente urbana, la cual, por cierto, "si bien ofrece oportunidades ocupacionales estacionales, padece graves problemas de servicios e infraestructura y se encuentra sujeta a fuertes contradicciones internas" (Velázquez, Tisnés y Gómez, 2014:6). Dichas características van acompañadas por un mismo origen, vinculado a la Ley $\mathrm{N}^{\circ} 9024 / 78$, la cual deviene de intereses políticos de fundar unidades subprovinciales separadas de gobiernos que priorizaban la actividad agropecuaria frente a la turística e inmobiliaria, dando lugar a la conformación de unidades espaciales hasta ese momento inéditas (Hernández, 2019). A la luz estos factores y los indicadores considerados en el IET, no debe llamar mucho la atención tal arrebatamiento del podio.

TABLA 5

Composición del IET en las regiones argentinas

\begin{tabular}{|c|l|l|l|l|l|l|}
\hline \multicolumn{2}{|c|}{} & \multicolumn{3}{|l|}{ IET $(\%)$} & \multicolumn{3}{l|}{} \\
\cline { 3 - 7 } \multicolumn{2}{|c|}{} & Alto & $\begin{array}{c}\text { Medio } \\
\text { Alto }\end{array}$ & Medio & $\begin{array}{c}\text { Medio } \\
\text { Bajo }\end{array}$ & Bajo \\
\hline \multirow{4}{*}{ REGION } & Pampeana* & 16,4 & 12,3 & 26,9 & 24,2 & 20,1 \\
\cline { 2 - 7 } & NEA** & 13,3 & 29,3 & 9,3 & 21,3 & 26,7 \\
\cline { 2 - 7 } & NOA**** & 24,2 & 22,2 & 12,1 & 16,2 & 25,3 \\
\cline { 2 - 7 } & Cuyo**** & 26,6 & 25,0 & 17,2 & 25,0 & 6,3 \\
\cline { 2 - 7 } & Patagonia***** & 28,3 & 28,3 & 18,9 & 5,7 & 18,9 \\
\hline
\end{tabular}

Fuente: elaboración personal

* Comprende Buenos Aires, CABA, Córdoba, Entre Ríos, La Pampa y Santa Fe

${ }^{* *}$ Comprende Chaco, Corrientes, Formosa y Misiones

*** Comprende Catamarca, Jujuy, Salta, Santiago del Estero y Tucumán

**** Comprende La Rioja, Mendoza, San Juan y San Luis

***** Comprende Chubut, Neuquén, Rio Negro, Santa Cruz y Tierra del Fuego

\section{Conclusiones}

El objetivo de este artículo ha sido dar a conocer el índice de especialización turística multidimensional aplicado sobre Argentina a escala departamental. Al tiempo que, en términos generales, el ranking turístico departamental ha mostrado coherencias, la distribución espacial del IET compuesta por un mosaico heterogéneo, pero con claras agrupaciones de la categoría superior sobre áreas de tradición turística como por ejemplo el Frente marítimo bonaerense, las Sierras cordobesas, la Patagonia andina y el Corredor termal entrerriano, también ha evidenciado el alcance de los procesos de fragmentación espacial relacionados con el turismo, aplicados al caso particular de Argentina.

Es importante señalar que la situación retratada representa a los espacios y tiempos previos al desencadenamiento de la pandemia por el covid-19, y que esta ha afectado al turismo externo e interno como ningún otro hecho. En relación a los venideros procesos de reapertura turística, contar con un indicador multidimensional como el IET puede ser de ayuda para la gestión y focalización política. No obstante, si bien esta primera aproximación ha dejado en evidencia que hay algunos datos disponibles, también ha plasmado las carencias existentes en materia de estadísticas turísticas departamentales, las cuales son indispensables si se 
quiere formular este tipo de herramientas. A pesar de todo, el resultado conseguido abre el camino a futuras exploraciones sobre cómo se relaciona el turismo con otras variables socioeconómicas desde el punto de vista espacial.

\section{REFERENCIAS}

Beier, L. S. (2015). Municipio turístico bonaerense: una propuesta de definición a partir de variables cuantitativas (Tesis de maestría en Desarrollo y Gestión del Turismo). Universidad Nacional de Quilmes, Argentina.

Benseny, G. (2006). El sector público turístico. Aportes sobre los organismos de turismo en la provincia de Buenos Aires - Argentina. Realidad, Tendencias y Desafíos en Turismo, 5(V), 109-133.

Bertoncello, R. (2006). Turismo, territorio y sociedad: el mapa turístico de la Argentina. En A. I. Geraiges de Lemos, M. Arroyo y M. L. Silveira (Orgs.), América Latina: cidade, campo e turismo (pp. 317-335). San Pablo: CLACSO.

Bertoncello, R. (Comp.) (2008). Turismo y geografia. Lugares y patrimonio natural-cultural de la Argentina. Buenos Aires. Ciccus.

Brau, R., Lanza, A., \& Pigliaru, F. (2007). How fast are small tourist countries growing? The 1980-2003 evidence. Recuperado de https://www.feem.it/m/publications_pages/NDL2007-001.pdf

Buzai, G. D. (2014). Mapas sociales urbanos. Buenos Aires: Lugar.

Callizo Soneiro, J. (1991). Aproximación a la Geografía del Turismo. Madrid: Síntesis.

Cazes, G. (2002). Turismo alternativo. En J. Jafari (Ed.), Enciclopedia del turismo (pp. 636-637). Madrid: Síntesis.

Cea D'Ancona, M. A. (1996). Metodología cuantitativa: estrategias y técnicas de investigación social. Madrid: Síntesis.

Fernández, M. R., Grill, D., y Laumann, Y. (2011). Relación entre el grado de especialización turística y el desarrollo económico para distintos países. Anuario Turismo y Sociedad, XII, 111-132.

González, R., Otero, A., Nakayama, L., y Marioni, S. (2009). Las movilidades del turismo y las migraciones de amenidad: problemáticas y contradicciones en el desarrollo de centros turísticos de montaña. Revista de Geografía Norte Grande, 44, 75-92.

Hernández, F. M. (2019). Estudio sobre la mercantilización de las playas en la costa marítima bonaerense. Estudios Socioterritoriales, 25(e017), 1-26.

Instituto Nacional de Estadísticas y Censos, INDEC (2013). Censo Nacional de Población, Hogares y Viviendas 2010. Base de datos Redatam. Definiciones de la base de datos. Recuperado de https://redatam.indec.gob.ar/redarg/ CENSOS/CPV2010rad/Docs/base.pdf

Lucero, P. (2016). El mapa social de Mar del Plata. Procesos de producción del espacio urbano y construcción de desigualdades territoriales (Tesis de doctorado en Geografía). Universidad Nacional del Sur, Departamento de Geografía y Turismo.

Mantero, J. C., Laffan, L., y Lefrou, D. (2011). Turismo residenciado- Turismo serviciado. Implicancias y alcances en Mar del Plata. Aportes y Transferencias, 15(II), 125-146.

Marchena Gómez, M. J. (2015). Gestión turística. En López Trigal (Dir.), Diccionario de geografía aplicada y profesional: terminologia de análisis, planificación y gestión del territorio pp. 297-298. León. Universidad de León.

Narodowski, P., Remes Lenicov, M., y Mallamace, M. (2014). El turismo. En P. Narodowski y M. Remes Lenicov (Coords.), Geografia Económica Mundial: un enfoque centro-periferia. Las cadenas globales de valor (Vol. II) (pp. 847-922). Moreno: UNM Editora.

Nogar, A. G., y Jacinto, G. P. (Comps.) (2010). Los espacios rurales. Aproximaciones teóricas y procesos de intervención en turismo rural. Buenos Aires. La Colmena.

Páez, S. G. A. (2013). Diferencias entre las relaciones matemáticas más usadas en demografía y geografía de la población. Revista Geográfica Venezolana, 54(2), 303-316.

Precedo Ledo, A., y Míguez Iglesias, A. (2007). La actividad turística como factor de desarrollo en el sistema español de asentamientos (1997-2002). Boletín de la A.G.E., 45, 191-211. 
Rodríguez, A. (2011). Calidad de vida y fragmentación socio-espacial en la provincia de Jujuy (República Argentina) en los inicios del siglo XXI. Investigaciones Geográficas, 43, 87-106.

Sánchez, D. C. (2011). Indicadores turísticos en la Argentina: una primera aproximación. Investigaciones Turisticas, 2, 29-65.

Sancho, A. (Dir.) (1998). Introducción al turismo. Madrid: OMT.

Schuschny, A., y Soto, H. (2009). Guí metodológica. Diseño de indicadores compuestos de desarrollo sostenible. Santiago de Chile: CEPAL.

Tapia, M., Quiroga, D., y Sánchez, D. (2015). La gran minería esinónimo de desarrollo?: la aplicación del índice de calidad de vida (ICV) en el caso de Antofagasta de la Sierra, provincia de Catamarca. RIEM, 12(VI), 41-66.

Velázquez, G. A. (2001). Geografía, Calidad de vida y fragmentación en la Argentina de los noventa. Análisis regional y departamental utilizando SIG's. Tandil: CIG.

Velázquez, G. A. (Dir.) (2016). Geografía y Calidad de vida en Argentina. Análisis regional y departamental (2010). Tandil: CIG.

Velázquez, G. A., y Celemín, J. P. (2013). La calidad ambiental en la Argentina: análisis regional y departamental (c.2010). Tandil: UNCPBA.

Velázquez, G. A., Tisnés, A., y Gómez, N. J. (2014). Región pampeana: Geografía y bienestar según subregiones (2010). Geograficando, 10(2), 1-26.

Vera Rebollo, J. F., Ponce Herrero, G. J., Dávila Linares, J. M., y Ramón Morte, A. (1990). Evaluación del grado de especialización turística de los municipios litorales valencianos. Investigaciones Geográficas, 8, 83-112.

Wallingre, N. (2014). Turismo, territorio y municipio. FACES, 20(42-43), 143-164.

\section{FuENTES DE DATOS}

INDEC (2002), Censo Nacional Agropecuario. Argentina

INDEC (2010), Censo Nacional de Población, Hogares y Viviendas. Argentina.

Sectur (2017). Registro de agencias de viajes. Argentina. 